\title{
The relationship between energy-adjusted dietary inflammatory index (E-DII) with quality of life and inflammatory markers among overweight and obese Iranian women: a cross-sectional study
}

\author{
Nasim Ghodoosi \\ Tehran University of Medical Sciences \\ Hana Arghavani \\ Tehran University of Medical Sciences \\ Atieh Mirzababaei \\ Tehran University of Medical Sciences \\ Mir Saeed Yekaninejad \\ Tehran University of Medical Sciences

\section{Seyed Ali Keshavarz} \\ Tehran University of Medical Sciences \\ Hossein Imani \\ Tehran University of Medical Sciences

\section{Farideh Shiraseb} \\ Tehran University of Medical Sciences \\ Khadijeh Mirzaei ( $\square$ mirzaei_kh@sina.tums.ac.ir) \\ https://orcid.org/0000-0002-7554-8551
}

\section{Research note}

Keywords: Energy-Adjusted dietary inflammatory index, overweight, Obesity, Quality of life, hs-CRP

Posted Date: August 24th, 2020

DOI: https://doi.org/10.21203/rs.3.rs-47521/v1

License: (c) (i) This work is licensed under a Creative Commons Attribution 4.0 International License. Read Full License 


\section{Abstract}

Objective: The objective of this study was to investigate associations between Energy-Adjusted Dietary Inflammatory Index (EDII) with quality of life and serum inflammatory markers.

Results: The mean age of the study participants was $36.30 \pm 8.05$ years. Analyses were performed using multivariable linear regression, adjusting for age, weight, physical activity, smoking status, economic status, and employment status. Linear regression analysis demonstrated that E-DII were significantly associated with certain the quality of life criteria, such as physical function, mental health, and vitality $(\beta=5.58,95 \% \mathrm{Cl} 0.72,10.43, p=$ $0.024, \beta=16.88,95 \% \mathrm{Cl} 10.75,23, \mathrm{p}<0.0001$ and $\beta=14.29,95 \% \mathrm{Cl} 9.48,20.36, \mathrm{p}<0.0001$, respectively). No significant associations were observed between EDII and serum level hs-CRP. It was found that dietary inflammatory potential decreased some quality of life measurements and levels in overweight and obese Iranian women.

\section{Introduction}

Obesity and overweight are some of the most prominent public health problems that mean excessive fat accumulation, defined as BMI (25-29.9) and BMI $\geq 30$ respectively, according to WHO definitions.[1, 2]. Overweight and obesity are common in Iran and are significantly more prevalent among women than men [3]. Accumulating evidence indicates that obesity is closely associated with an increased risk of cardiovascular disease (CVD), hypertension (HTN), type 2 diabetes mellitus (T2DM), strokes, and poor health quality of life (HQOL) [4].

In addition to medical complications, overweight and obesity is accompanied by a decline in health-related quality of life factors, such as physical functioning, psychosocial functioning, and emotional well-being [5-8]. The concept of quality of life (QoL) is a complex, multifaceted construct that includes various aspects, such as physical health and psychological health [9].

The results of recent studies show that obese people with lower BMI have higher quality of life than those with higher BMI scores [10]. The rising prevalence and health-related consequences of obesity make it a public health concern all over the world [11, 12]. Furthermore, obesity is typically associated with a chronic state of systemic low-grade inflammation since adipocytes result in the expression of cytokines such as Hypersensitive serum Creactive protein (hs-CRP) $[13,14]$. Recent studies have shown reliable associations between diet and systemic inflammation so that diet can play a major and significant role in quality of life[15, 16]. The energy-adjusted dietary inflammatory index is a validated method developed to characterize dietary inflammatory potential [17]. Given all the above, it was hypothesized that greater adherence to an anti-inflammatory diet would be associated with lower low-grade inflammation and greater quality of life in overweight and obese patients. To the researchers' knowledge, this is the first study investigating the relationship between energy-adjusted dietary inflammatory index and quality of life.

\section{Methods}

\section{Study Design and Participants}

The current cross-sectional study was conducted among overweight and obese women who attended health centers in Tehran, Iran, in 2018. A random sample of 280 adult healthy overweigh and obese aged between 18 and 
50 years old women was selected from 20 various health centers by a multistage cluster random sampling method. Eligible criteria included body mass index in the range of $25-40 \mathrm{~kg} / \mathrm{m}^{2}$ and good general health. Exclusion criteria included: history of cardiovascular disease, hypertension, diabetes mellitus, hepatic or renal disease, and alcohol, smoking usage, medicine usage other than birth control pills, pregnancy or lactation, menopause, following a specific diet or body weight fluctuation over the past 1 year.

Participants whose reported daily energy intake less than $800 \mathrm{kcal} / \mathrm{d}$ or more than $4200 \mathrm{kcal} / \mathrm{d}$ were also excluded. Informed consent was obtained from all participants.

\section{Dietary Assessment}

Participants' dietary intake over the past year was assessed using a valid and reliable semi-quantitative food frequency questionnaire (FFQ) self-reported[18]. This FFQ consists of 147 food items with standard serving sizes, and participants were asked to specify their consumption frequency for each food item. The consumed foods portion sizes were converted to grams using household measurements[19]. Then, nutrient and energy intakes were computed using NUTRITIONIST IV software (version 7.0; N-Squared Computing, Salem, OR), which was tailored for Iranian foods. For calculating EDII, all nutrient values were adjusted for energy intake using the residual method.

\section{Dietary Inflammatory Index Calculation}

To calculate EDII for the participants of this study, the dietary data were first linked to the regionally representative world database, which provided a robust estimate of a mean and standard deviation for each parameter. These then become the multipliers to express an individual's exposure relative to the 'standard global mean' as a z-score. A z-score for each food consumed was calculated by subtracting the 'standard mean' from the actual food parameter value and divided by its standard deviation. Next, to minimize the effect of 'right skewing', this value was then converted to a centered percentile score, which was then multiplied by the respective food parameter inflammatory effect score to obtain the subject's food parameter-specific EDII score. All of the food-parameterspecific EDII scores were then summed together to create an overall EDII score for every subject in the study [20]. In total, the EDIl computed based on this study's FFQ includes data on 29 of the 45 possible food variables composing the EDIl: energy, carbohydrate, protein, fat, fiber, cholesterol, trans fat, SFAs, MUFAs, PUFAs, omega-3, omega-6, niacin, thiamin, riboflavin, vitamin B-6, vitamin B-12, iron, magnesium, selenium, zinc, vitamin A, vitamin C, vitamin D, vitamin E, folic acid, b-carotene, caffeine, onion and tea.

\section{Quality of Life Assessment}

The SF-36 is a short-form, self-administered quality of life scoring questionnaire. It consists of 36 questions, 35 of which are compressed into eight multi-item scales including physical functioning (PF), role physical (RP), bodily pain (BP), general health (GH), vitality (VT), social functioning (SF), role emotional (RE), and mental health (MH) $[21,22]$. The SF-36 also includes a question self-evaluating health changes in the past year (reported health), which does not belong to the eight dimensions, or the total SF-36 score. Each of these 8 dimensions has a score between 0 (worst health) to 100 (best health). [23-25]

\section{Biochemical Assessment}


Blood samples were collected early in the morning after 12-hour night-time fasting at the Nutrition and the Biochemistry Laboratory of the School of Nutritional Sciences Dietetics, TUMS. Samples of blood collected in parent tubes containing 0.1 EDTA, was taken in accordance with the standard protocol in a sitting position. Samples of serum were centrifuged for serum collection $10 \mathrm{~min}$ at $300 \mathrm{rpm}$, diluted in $1 \mathrm{ml}$ tubes, and stored at $-80^{\circ} \mathrm{C}$ until the analysis. Tests were analyzed utilizing the Auto-Analyzer BT 1500 (Selectra 2; Vital Scientific, Spankeren, Netherlands). Hypersensitive serum C-reactive protein levels were measured by an immunoturbidimetric test with the Pars Azmoon kit (Pars Azmoon Inc. Tehran, Iran).

\section{Anthropometric Assessment}

Anthropometric measures, including body weight, body mass index, waist circumferences, and waist-hip ratio, were measured in an overnight fasting state, without shoes, with minimal clothing and by the use of a multi-frequency bioelectrical impedance analyzer In-body 770 scanner by trained dietitians (In-body Co., Seoul, Korea). Height was measured with a wall Seca 206 stadiometer (Hamburg, Germany), based on a standard protocol, their height was recorded to the nearest $0.2 \mathrm{~cm}$.

\section{Assessment of other Variables}

\section{Statistical Analyses}

The normality of variables distribution was checked using the Kolmogorov-Smirnov test. we had no missing data. The EDII was analyzed as a dichotomous variable, categorized based on the median value of the EDII (0.05). EDII (as dichotomous) was examined across the following characteristics: age, weight, height, economic status, BMI, waist circumference, waist-hip ratio, energy intake, physical activity, and quality of life measurements, via independent sample T-test analyses. Comparisons of different food group intakes across the EDII quartiles were analyzed through an independent sample T-test. Multivariable linear regression analyses of the continuous EDII score were conducted to determine the association of the EDII with quality of life and hs-CRP levels. Variables were adjusted for the following confounding factors: age, weight, physical activity, smoking, economic status, and employment status. The results are reported as percentage change $(\beta)$ with $95 \%$ confidence intervals $(95 \% \mathrm{Cl})$. Statistical analysis was performed using SPSS version 21 (SPSS Inc., Chicago, USA). Significance was set at a probability of $\leq 0.05$ for all tests.

\section{Results}

The dietary inflammatory potential scores in this study, as measured by EDII, ranged from -4.14 (most antiinflammatory score) to 3.89 (most pro-inflammatory score). The mean (SD) age of the participants at recruitment was 36 (8). Table 1 presents participants' characteristics in relation to different categories of dietary inflammatory indices. EDII was categorized into anti-inflammatory (EDII $\leq 0 / 05)$ and pro-inflammatory (EDII $>0 / 06)$ diets, based on the median value (0.05). When EDII was converted into two groups, significant differences were observed for IPAC $(\mathrm{P}<0.0001)$. The hs-CRP level showed no significant change in the higher EDIl score group compared to the other group (PC: $4 / 26 \pm 4 / 42 \%$ vs. $4 / 07 \pm 4 / 34 \% ; P=0.856$ ).

\section{Table 1. Participant characteristics between EDII groups}




\begin{tabular}{|c|c|c|c|c|}
\hline Participant Characteristics & EDII Groups & Mean & Std. Deviation & $P$ value \\
\hline \multirow[t]{2}{*}{ Age(year) } & $<=.05$ & 37.60 & 7.58 & \multirow[t]{2}{*}{0.105} \\
\hline & $.06+$ & 35.03 & 8.48 & \\
\hline \multirow[t]{2}{*}{ Weight(kg) } & $<=.05$ & 81.09 & 12.56 & \multirow[t]{2}{*}{0.317} \\
\hline & $.06+$ & 80.61 & 12.09 & \\
\hline \multirow[t]{2}{*}{ Height(cm) } & $<=.05$ & 161.25 & 6.24 & \multirow[t]{2}{*}{0.217} \\
\hline & $.06+$ & 161.33 & 5.75 & \\
\hline \multirow[t]{2}{*}{$\mathrm{BMI}\left(\mathrm{kg} / \mathrm{m}^{2}\right)$} & $<=.05$ & 30.94 & 3.99 & \multirow[t]{2}{*}{0.130} \\
\hline & $.06+$ & 30.52 & 3.56 & \\
\hline \multirow[t]{2}{*}{ Waist Circumference(cm) } & $<=.05$ & 99.25 & 10.24 & \multirow[t]{2}{*}{0.391} \\
\hline & $.06+$ & 98.97 & 9.89 & \\
\hline \multirow[t]{2}{*}{ Waist-Hip Ratio } & $<=.05$ & .93 & .05 & \multirow[t]{2}{*}{0.651} \\
\hline & $.06+$ & .93 & .05 & \\
\hline \multirow[t]{2}{*}{ Energy Intake(kcal) } & $<=.05$ & 2588.82 & 707.15 & \multirow[t]{2}{*}{0.140} \\
\hline & $.06+$ & 2658.21 & 790.42 & \\
\hline \multirow[t]{2}{*}{ IPAC(MET-min/week) } & $<=.05$ & 1162.45 & 1322.88 & \multirow[t]{2}{*}{$<0.0001$} \\
\hline & $.06+$ & 773.24 & 692.04 & \\
\hline \multirow[t]{2}{*}{ Hs-CRP(mg/L) } & $<=.05$ & 4.26 & 4.42 & \multirow[t]{2}{*}{0.856} \\
\hline & $.06+$ & 4.07 & 4.34 & \\
\hline \multirow[t]{2}{*}{ SF Total } & $<=.05$ & 77.32 & 13.43 & \multirow[t]{2}{*}{0.715} \\
\hline & $.06+$ & 72.11 & 12.80 & \\
\hline
\end{tabular}

$n=280$

Data are presented as mean \pm standard deviation.

Nutrients intake adjusted for energy intake before calculating EDII

DII values were categorized according to the median

(DII $\leq$ 0/05: Anti-inflammatory diet, DII > 0/06: pro-inflammatory diet)

Independent sample $t$ test was used for comparison of continuous variables between DII categories 
Significant inverse differences were found between the three dimensions of SF-36, including physical functioning $(P=0.033)$, mental health $(P=0.021)$, and vitality $(P=0.031)$, with anti-inflammatory and pro-inflammatory diet groups. For the other five dimensions, such as general health, role-physical, role emotional, social functioning, bodily pain, and health transition, decreasing trends were observed across EDIl categories, but these relationships were not significant (Table 2).

Table 2. Quality of life items by level of EDII groups

\begin{tabular}{|c|c|c|c|c|}
\hline SF-36 Items & DII Groups & Mean & Std. Deviation & P-value \\
\hline \multirow[t]{2}{*}{ General Health } & $<=.05$ & 68.47 & 18.88 & 0.082 \\
\hline & $.06+$ & 66.92 & 16.77 & \\
\hline \multirow[t]{2}{*}{ Physical Functioning } & $<=.05$ & 84.37 & 16.65 & 0.033 \\
\hline & $.06+$ & 79.16 & 22.33 & \\
\hline \multirow[t]{2}{*}{ Role Physical } & $<=.05$ & 91.24 & 28.37 & 0.094 \\
\hline & $.06+$ & 88.15 & 32.44 & \\
\hline \multirow[t]{2}{*}{ Role Emotional } & $<=.05$ & 86.78 & 33.89 & 0.073 \\
\hline & $.06+$ & 82.89 & 37.71 & \\
\hline \multirow[t]{2}{*}{ Social Functioning } & $<=.05$ & 73.65 & 22.71 & 0.968 \\
\hline & $.06+$ & 70.76 & 23.53 & \\
\hline \multirow[t]{2}{*}{ Bodily Pain } & $<=.05$ & 62.31 & 21.02 & 0.871 \\
\hline & $.06+$ & 63.09 & 20.24 & \\
\hline \multirow[t]{2}{*}{ Vitality } & $<=.05$ & 76.37 & 18.35 & 0.031 \\
\hline & $.06+$ & 60 & 22.28 & \\
\hline \multirow[t]{2}{*}{ Mental Health } & $<=.05$ & 82.33 & 20.82 & 0.021 \\
\hline & $.06+$ & 66.28 & 24.52 & \\
\hline \multirow[t]{2}{*}{ Health Transition Item } & $<=.05$ & 51.82 & 29.34 & 0.056 \\
\hline & $.06+$ & 46.67 & 25.70 & \\
\hline \multirow[t]{2}{*}{ SF-36 -TOTAL } & $<=.05$ & 77.32 & 13.43 & 0.715 \\
\hline & $.06+$ & 72.11 & 12.80 & \\
\hline
\end{tabular}

$n=280$

Data are presented as mean \pm standard deviation.

Nutrients intake adjusted for energy intake before calculating EDII 
EDII values were categorized according to the median.

(Anti-inflammatory diet: EDII $\leq$ 0/05, pro-inflammatory diet: EDII >0/06)

Independent sample t-test was used for comparison of continuous quality of life measures between EDII categories

Table 3 shows the distribution of 11 food groups: grains, refined grains, dairy products, beans, vegetables, fruits, red meat, processed meat, white meat, nut/olive group and sweet/fat group across the EDII groups. The results showed that servings of vegetables and beans $(P<0.0001)$ decreased significantly in the higher dietary inflammatory index group, whereas servings of sweet/fat group $(P<0.0001)$ increased significantly in this group.

Table 3. Relationships between servings of food groups across EDII groups 


\begin{tabular}{|c|c|c|c|c|}
\hline Food Groups(g/day) & EDII Groups & Mean & Std. Deviatior & $P$ value \\
\hline \multirow[t]{2}{*}{ Grains } & $<=.05$ & 458.59 & 218.60 & \multirow[t]{2}{*}{0.749} \\
\hline & $.06+$ & 507.98 & 242.73 & \\
\hline \multirow[t]{2}{*}{ Refined Grains } & $<=.05$ & 385.37 & 220.20 & \multirow[t]{2}{*}{0.274} \\
\hline & $.06+$ & 479.92 & 243.59 & \\
\hline \multirow[t]{2}{*}{ Dairy Products } & $<=.05$ & 390.78 & 217.44 & \multirow[t]{2}{*}{0.272} \\
\hline & $.06+$ & 351.84 & 270.79 & \\
\hline \multirow[t]{2}{*}{ Beans } & $<=.05$ & 57.75 & 49.82 & \multirow[t]{2}{*}{$<0.0001$} \\
\hline & $.06+$ & 41.01 & 26.91 & \\
\hline \multirow[t]{2}{*}{ Vegetables } & $<=.05$ & 535.37 & 250.44 & \multirow[t]{2}{*}{$<0.0001$} \\
\hline & $.06+$ & 292.86 & 193.06 & \\
\hline \multirow[t]{2}{*}{ Fruits } & $<=.05$ & 653.14 & 354.93 & \multirow[t]{2}{*}{0.060} \\
\hline & $.06+$ & 454.64 & 326.29 & \\
\hline \multirow[t]{2}{*}{ Red Meat } & $<=.05$ & 46.45 & 24.31 & \multirow[t]{2}{*}{0.995} \\
\hline & $.06+$ & 42.80 & 25.40 & \\
\hline \multirow[t]{2}{*}{ Processed Meat } & $<=.05$ & 4.08 & 10.48 & \multirow[t]{2}{*}{0.862} \\
\hline & $.06+$ & 5.65 & 7.92 & \\
\hline \multirow[t]{2}{*}{ White Meat } & $<=.05$ & 50.27 & 40.06 & \multirow[t]{2}{*}{0.987} \\
\hline & $.06+$ & 43.71 & 51.31 & \\
\hline \multirow[t]{2}{*}{ Nut.Olive } & $<=.05$ & 13.87 & 11.52 & \multirow[t]{2}{*}{0.116} \\
\hline & $.06+$ & 9.94 & 10.93 & \\
\hline \multirow[t]{2}{*}{ Sweet. Fat } & $<=.05$ & 80.27 & 50.13 & \multirow[t]{2}{*}{$<0.0001$} \\
\hline & $.06+$ & 126.26 & 111.59 & \\
\hline
\end{tabular}

$n=280$

Data are presented as mean \pm standard deviation.

Nutrients intake adjusted for energy intake before calculating EDII

EDIl values were categorized according to the median.

(EDII $\leq$ 0/05: Anti-inflammatory diet, EDII > 0/06: Pro-inflammatory diet)

Independent sample $t$ test was used for comparison of food group's intake between EDII 
A direct association between EDII and physical functioning levels was observed after adjustment for potential confounders, such as age, weight, smoking, economic status, and employment status ( $P=0.024)$ (shown in Additional file1: Table S1).

Multivariable linear regression analysis with adjustments for potential confounders demonstrated that EDII were significantly associated with mental health and vitality $(\beta=5.58,95 \% \mathrm{Cl} 0.72,10.43, \mathrm{p}=0.024, \beta=16.88,95 \% \mathrm{Cl}$ 10.75, 23, $\mathrm{p}<0.0001$ (shown in Additional file2: Table S2)and $\beta=14.29,95 \% \mathrm{Cl} 9.48,20.36, \mathrm{p}<0.0001$ (shown in Additional file3: TableS3), respectively.

\section{Discussion}

It is important to note that this is the first study to examine the association between dietary inflammatory potential, quality of life and inflammation levels among women. This cross-sectional study of Iranian women showed evidence of a positive association between higher EDII scores with a lower quality of life in certain measurements, such as physical functioning, mental health, and vitality. These findings emphasize the importance of addressing overall dietary quality in future community- or population-based programs or policies to prevent chronic disease.

Some prior research has shown that an inflammatory diet plays an important role in psychological health.

Almudena Sánchez-Villegas et al. also determined that a pro-inflammatory diet was associated with a significantly higher risk of depression in a Mediterranean population, particularly in older subjects [28]. Tasnime N. Akbaraly et al. found that a pro-inflammatory diet was associated with recurrent depression in women, which seems not to be driven by circulating inflammatory markers [29]. There is considerable evidence that has suggested a better quality of diet or an anti-inflammatory diet is associated with better quality of life and mental health. Ujué Fresán et al. have found that a Mediterranean diet was associated with reduced depression risks [30]. The current findings are supported by an Australian study which reported that adherence to an MD pattern and better dietary quality is associated with better mental health and wellbeing [31, 32]. A possible mechanism seems to related to the fact that an anti-inflammatory diet is rich in nutrients such as vitamins, minerals, antioxidants, and fiber, which have beneficial health effects that have been widely demonstrated.

The results revealed no relationship between EDII and hs-CRP levels in women. To the best of the researchers' knowledge, previous studies of dietary inflammatory indices and serum hs-CRP levels are generally consistent with the findings of the present study. Several other studies have suggested that a pro-inflammatory diet is associated with higher levels of inflammatory markers. Previous studies have shown that DII was associated with an increase in the odds of elevated hs-CRP levels (.3 mg/l) [33]. Another study in the USA demonstrated that higher DII scores were associated with inflammatory biomarkers including IL-6, TNF-a, and hs-CRP [34]. In the Asklepios study, no significant associations were observed between DII and CRP and fibrinogen, but significant positive associations between DII and IL-6 and homocysteine were observed [35]. Utilizing DII is one of the strengths of the present investigation. DIl found Overall diet, rather than individual foods and nutrients.

\section{Conclusion}


The most interesting finding of this study is that more anti-inflammatory diets are significantly associated with higher physical function, mental health, and vitality in overweight and obese women. Thus, encouraging the intake of more anti-inflammatory dietary factors, such as plant-based foods rich in fiber and phytochemicals, and reducing intake of pro-inflammatory factors, such as fried foods or processed foods rich in saturated fat, maybe a beneficial strategy for better physical and mental health.

\section{Limitations}

The present study had some limitations that should be considered. First of all, the use of self-reported FFQ is known to contain a certain degree of measurement error, which might affect results. Moreover, an FFQ consisting of 29 instead of 45 food parameters was used for calculating EDII. The small sample size is another limitation, and data from larger sample size studies are more credible. Because of our study population that was 18-50 years old, overweight, and obese women, the relationship could not be generalizable to other populations of different ages, genders, and backgrounds. Besides, since the research has a cross-sectional study, the causal relationship between energy-adjusted dietary inflammatory index, inflammatory markers, and quality of life cannot be inferred using retrospective observational studies; conducting prospective studies is thus highly recommended

\section{Abbreviations}

BMI: Body mass index, CVD: Cardiovascular disease; CRP: C-reactive protein; EDII: Energy-Adjusted dietary inflammatory index; ELISA: Enzyme-linked immunosorbent assay; FFQ: Food frequency questionnaire; HQOL: Health quality of life; HTN: Hypertension; Hs-CRP: High-sensitivity C-reactive protein; IL-6: Interleukin-6; IPAQ: International Physical Activity Questionnaires; MUFA: Monounsaturated fatty acids; METs: Metabolic Equivalent Tasks; PUFA: Polyunsaturated fatty acids; QOL: Quality of life; SFA: Saturated fatty acids; T2DM: Type 2 diabetes mellitus; TNFa: Tumor necrosis factor-a; WHO: World Health Organization.

\section{Declarations}

\section{Ethics approval and consent to participate}

The study protocol has approved by the ethics committee of Endocrinology and Metabolism Research Center of Tehran University of Medical Sciences (TUMS) with the following identification: IR.TUMS.VCR.REC.1395.1597. Each participant was completely informed about the study protocol and provided a written and informed consent form before taking part in the study. This study was approved by the research council (research project number: 95-03-161-33142, 95-04-161-33893).

\section{Consent for publication}

Each participant was completely informed about the study protocol and provided a written and informed consent form before taking part in the study.

\section{Availability of data and materials}


The data that support the findings of this study are available from Khadijeh Mirzaei but restrictions apply to the availability of these data, which were used under license for the current study, and so are not publicly available. Data are however available from the authors upon reasonable request and with permission of Khadijeh Mirzaei.

\section{Competing interests}

The authors declare that they have no competing interests.

\section{Funding}

This study was supported by grants from the Tehran University of Medical Sciences, Tehran, Iran (Grant number: 95-03-161-33142, 95-04-161-33893).

\section{Authors' contributions}

The project was designed and implemented by NGh and KhM. Data were analyzed and interpreted NGH, HA, AM, SY, SAK, FSH, and HI prepared the manuscript. KhM, supervised the overall project. All authors read and approved the final manuscript.

\section{Acknowledgements}

We thank the School of Public Health, Tehran University of Medical Sciences and we would like to thank all the participants for their cooperation.

\section{Author details}

${ }^{1}$ Department of Community Nutrition, School of Nutritional Sciences and Dietetics, Tehran University of Medical Sciences (TUMS), P.O. Box: 14155-6117, Tehran, Iran. ${ }^{2}$ Department of Epidemiology and Biostatistics, School of Public Health, Tehran University of Medical Sciences (TUMS), Tehran, Iran. ${ }^{3}$ Department of Clinical Nutrition, School of Nutritional Sciences and Dietetics, Tehran University of Medical Sciences.

\section{References}

1. Gallus, S., et al., Overweight and obesity prevalence and determinants in Italy: an update to 2010. European journal of nutrition, 2013. 52(2): p. 677-685.

2. Pietrzyk, L., et al., Obesity and obese-related chronic low-grade inflammation in promotion of colorectal cancer development. Asian Pac J Cancer Prev, 2015. 16(10): p. 4161-4168.

3. Ayatollahi, S. and Z. Ghoreshizadeh, Prevalence of obesity and overweight among adults in Iran. Obesity reviews, 2010. 11(5): p. 335-337. 
4. Williams, E.P., et al., Overweight and obesity: prevalence, consequences, and causes of a growing public health problem. Current obesity reports, 2015. 4(3): p. 363-370.

5. Kushner, R.F. and G.D. Foster, Obesity and quality of life. Nutrition, 2000. 16(10): p. 947-52.

6. Fontaine, K.R. and I. Barofsky, Obesity and health-related quality of life. Obes Rev, 2001. 2(3): p. $173-82$.

7. Kolotkin, R.L., K. Meter, and G.R. Williams, Quality of life and obesity. Obes Rev, 2001. 2(4): p. $219-29$.

8. Sullivan, M., et al., Why quality of life measures should be used in the treatment of patients with obesity. International Textbook of Obesity. New York: John Wiley \& Sons, 2001: p. 485-510.

9. Taylor, V.H., et al., The impact of obesity on quality of life. Best practice \& research Clinical endocrinology \& metabolism, 2013. 27(2): p. 139-146.

10. Pimenta, F.B., et al., The relationship between obesity and quality of life in Brazilian adults. Frontiers in psychology, 2015. 6.

11. Kelly, T., et al., Global burden of obesity in 2005 and projections to 2030. International journal of obesity, 2008. 32(9): p. 1431.

12. Ng, M., et al., Global, regional, and national prevalence of overweight and obesity in children and adults during 1980-2013: a systematic analysis for the Global Burden of Disease Study 2013. The lancet, 2014. 384(9945): p. 766-781.

13. Kim, C., et al., Circulating levels of MCP-1 and IL-8 are elevated in human obese subjects and associated with obesity-related parameters. International journal of obesity, 2006. 30(9): p. 1347.

14. Singer, K. and C.N. Lumeng, The initiation of metabolic inflammation in childhood obesity. The Journal of clinical investigation, 2017. 127(1): p. 65-73.

15. Costarelli, V., E. Koretsi, and E. Georgitsogianni, Health-related quality of life of Greek adolescents: the role of the Mediterranean diet. Quality of life research, 2013. 22(5): p. 951-956.

16. Veronese, N., et al., Adherence to the Mediterranean diet is associated with better quality of life: data from the Osteoarthritis Initiative. The American journal of clinical nutrition, 2016. 104(5): p. 1403-1409.

17. Wirth, M., et al., Association of a Dietary Inflammatory Index with Inflammatory Indices and the Metabolic Syndrome among Police Officers. Journal of occupational and environmental medicine/American College of Occupational and Environmental Medicine, 2014. 56(9): p. 986.

18. Mirmiran, P., F. Hosseini Esfahani, and F. Azizi, Relative validity and reliability of the food frequency questionnaire used to assess nutrient intake: Tehran Lipid and Glucose Study. Iran J Diabetes Lipid Disord, 2009. 9: p. 185-97.

19. Ghaffarpour, M., A. Houshiar-Rad, and H. Kianfar, The manual for household measures, cooking yields factors and edible portion of foods. Tehran: Nashre Olume Keshavarzy, 1999. 7: p. 213.

20. Shivappa, N., et al., Designing and developing a literature-derived, population-based dietary inflammatory index. Public health nutrition, 2014. 17(8): p. 1689-1696.

21. McHorney, C.A., J.E. Ware Jr, and A.E. Raczek, The MOS 36-Item Short-Form Health Survey (SF-36): II. Psychometric and clinical tests of validity in measuring physical and mental health constructs. Medical care, 1993: p. 247-263.

22. Kalantar-Zadeh, K., et al., Association among SF36 quality of life measures and nutrition, hospitalization, and mortality in hemodialysis. Journal of the American Society of Nephrology, 2001. 12(12): p. 2797-2806. 
23. Brazier, J.E., et al., Validating the SF-36 health survey questionnaire: new outcome measure for primary care. Bmj, 1992. 305(6846): p. 160-164.

24. Arefnasab, Z., et al., Effect of mindfulness based stress reduction on quality of life (SF-36) and spirometry parameters, in chemically pulmonary injured veterans. Iranian journal of public health, 2013. 42(9): p. 1026.

25. Lins, L. and F.M. Carvalho, SF-36 total score as a single measure of health-related quality of life: Scoping review. SAGE open medicine, 2016. 4: p. 2050312116671725.

26. Craig, C.L., et al., International physical activity questionnaire: 12-country reliability and validity. Medicine and science in sports and exercise, 2003. 35(8): p. 1381-1395.

27. Committee, I.R., Guidelines for data processing and analysis of the International Physical Activity Questionnaire (IPAQ)-short and long forms. http://www. ipaq. ki. se/scoring. pdf, 2005.

28. Sánchez-Villegas, A., et al., Dietary inflammatory index, cardiometabolic conditions and depression in the Seguimiento Universidad de Navarra cohort study. British Journal of Nutrition, 2015. 114(9): p. 1471-1479.

29. Akbaraly, T.N., et al., Dietary inflammatory index and recurrence of depressive symptoms: Results from the Whitehall II Study. Clinical Psychological Science, 2016. 4(6): p. 1125-1134.

30. Fresán, U., et al., Does the MIND diet decrease depression risk? A comparison with Mediterranean diet in the SUN cohort. European journal of nutrition, 2018: p. 1-12.

31. Bonaccio, M., et al., Adherence to a Mediterranean diet is associated with a better health-related quality of life: a possible role of high dietary antioxidant content. BMJ open, 2013. 3(8): p. e003003.

32. Milte, C.M., et al., Associations of diet quality with health-related quality of life in older Australian men and women. Experimental gerontology, 2015. 64: p. 8-16.

33. Shivappa, N., et al., A population-based dietary inflammatory index predicts levels of C-reactive protein in the Seasonal Variation of Blood Cholesterol Study (SEASONS). Public health nutrition, 2014. 17(8): p. 1825-1833.

34. Tabung, F.K., et al., Construct validation of the dietary inflammatory index among postmenopausal women. Annals of epidemiology, 2015. 25(6): p. 398-405.

35. Shivappa, N., et al., Associations between dietary inflammatory index and inflammatory markers in the Asklepios Study. British Journal of Nutrition, 2015. 113(4): p. 665-671.

\section{Supplementary Files}

This is a list of supplementary files associated with this preprint. Click to download.

- Additionalfile3TableS3.docx

- Additionalfile2Tables2.docx

- Additionalfile1Tables1.docx 